\title{
State and Space Vectors - Complementary Description of the 2-level VSI
}

\author{
J. Iwaszkiewicz, L. Wolski \\ The Electrotechnical Institute, Gdansk Branch \\ Narwicka 1, 80-557 Gdansk (Poland) \\ Phone/Fax number:+48 583431291, e-mail: jan.iwaszkiewicz@iel.gda.pl, leszek.wolski@iel.gda.pl
}

\begin{abstract}
.
The paper deals with a generalized description system of the two level inverter. Two methods of mathematical approach to the representation of the converter's state are presented and discussed. One method is standard space vector and the other, originally designed by authors, is state vector representation. The notational system provides a general simplification and standardization of vectors identification. It contains a novel handy proposal of notation and marking which is useful for converter description. The notation system permits to accomplish a comparison and correlation between space and state vectors. It describes state and space vectors by use of the same numbers. Thanks to these properties the proponed mathematical tool can be extremely useful in designing the control algorithms for easy implementation and fast performance of the converter
\end{abstract}

\section{Key words}

two level converter control, converter model, control algorithm, state vector, space vector.

\section{Introduction}

A great demand for electric energy converters is accompanying modernization and development processes in contemporary industry and public area. The increasing area of requirements as well as volume of energy has got a great impact on application and development of inverters. The suitable inverters should be able to supply diverse equipment working in a large power range from less than one to hundreds $\mathrm{kW}$ and more. Thus the converters have to fulfill specific and diversified requirements what implies different methods of electric energy conversion. For instance in AC drives where the ability of speed regulation is essential, the converter, e.g. a voltage or current source inverter (VSI or CSI), has to assure the adjustment of output voltage fundamental and frequency [1]. Evidently the application area of inverters is very large and there are many other industrial applications e.g. uninterruptible power supplies (UPS) or distributed power generation systems, where the essential demand is to generate 50 or $60 \mathrm{~Hz}$ sinusoidal voltage waveforms. Even if there is a need of the output waveform adjustment, the output parameters have to be attuned in a relatively small range. In numerous devices such as AC drives, UPSs, active filters or voltage regulators in electric grids the main and most important features are the following: high quality of output waveforms, output stability and efficiency of the device [2],[3]. Similar requirements are to be fulfilled in converters applied in renewable energy systems as wind farms or static DC sources in photovoltaic farms and fuel cells. The same expectations are concerning converters working in numerous applied systems of power quality improvements. A lot of power quality and renewable energy systems require application of medium or even lower power range converters. This power range could be satisfactory covered by two level inverters

During first period of power electronics evolution the two-level inverter became the most suitable device used to energy conversion in aforesaid applications. Its features and performance as well as drawbacks have been largely recognized and verified in practice [4]. The range of the output power is determined by semiconductor switches properties and is an essential limitation of the two-level inverter. Although latest achievements in power semiconductors technology permit to work with elevated power and frequency but fast switching accompanying the PWM control causes power losses in switching elements resulting in cutting the inverter efficiency down. All these reasons and drawbacks brought into significant development of multilevel inverters. Thanks to their promising performance, multilevel inverters are able to reduce the essential disadvantages of two-level inverters. First of all they permit to overcome the problem of limited power and to shape perfect output waveforms. But two level inverters are used in many applications where their properties are sufficient.

The development of two and multilevel converters still comprises a research area for new topologies, control strategies and theory. The complexity of converters' structures, number of semiconductor switches and reachable vectors lead to use of extremely sophisticated control methods and algorithms. This requires availability of good mathematical tools and converter models which are useful to the purpose of control algorithm composition 
and guarantee easy implementation and fast performance of the converter.

In subject literature one can find a great differentiation of proposals concerning converter models, systems of switching states notation and mathematical tools that authors use for solving this task [5].

In [6] the authors present one sector of the space vector diagram of a five level inverter. The obtainable vectors are denoted by use of indices $0,1, \ldots 14$ : A0, A1,..A14 while the converter states by numbers: $-2,-1,0,1,2$. According to the assumed notation system the vector A10 can be expressed as (2-2-2) and A14 as (22-2). All multiple vectors have the same index e.g. A0 (-2-2-2), (-1-1-1), (000), (111), (222), A1 (-1-2-2), (0-1-1), (100), (211) and so on. So the vectors can be written by use of only three alphanumeric signs (111), four as (22-2), five (2-2-2) and six (-2-2-2). In order to select one of multiple vectors, the most optimal in real converter state, it is necessary to introduce additional procedures to the control algorithm. An evident tendency to signify space vectors by use of additional signs "+" and "-." or even by letters "P" and " $\mathrm{N}$ " is noticeable in many systems of multilevel inverter circumscription [7], [8],[9],[10].

A majority of researchers assume as more useful the notation systems in which space voltage vectors are denoted by numbers. An example presented in [11] illustrates vectors of a five-level three-phase inverter denoted by numbers $(c b a)$. The number expansion consists of three digits corresponding to the voltage level per phase. Thus, the vector number 400 corresponds to the voltage level 4 in phase $a$ and voltage level 0 in phases $b$ and $c$. This notational system could be also useful for defining some states of fault operation on multilevel inverters [12]. Evidently the systems based on numbers (generally on whole numbers) are largely applied in many variants and according to divers notation order.

When the number of voltage levels increases the number of obtainable vectors is quickly rising and for a 7level inverter reaches 343 vectors [13]. Usually inverter vectors are identified by use of indices e.g. $\mathrm{V}_{1}, \mathrm{~V}_{2}, \ldots$ and in general the indices represent decimal numbers. The correlation between vector numbers and converter states is often very poor. Moreover it happens that the notational system has been assumed casually or has no mnemonic features. It would be desirable that the notational system was based on symbols or numbers which are in accordance to selected rules and in relation with fundamental elements of the converter model, more particularly related to the inverter switching states. Although there are many proposals for this purpose but there is no one canon respected and accepted in this matter [14], [15].

The paper deals with a description system of the 2level inverter. The notational system provides a general simplification and standardization of vectors identification. It contains a novel handy proposal of notation and marking which is useful for converter description as well as producing control algorithms. The notation system permits to accomplish a comparison and correlation between space and state vectors. It describes state and space vectors by use of the same numbers. The main rule of the presented system can be easily enlarged on multilevel inverters.

\section{Two Level Voltage Source Inverter: State and Space Vectors}

\section{A. Two-Level Inverter Model}

A simplified model of the two-level inverter is presented in Fig. 1. The model consists of three two-state switches $K a, K b, K c$ assigned accordingly to output phases $a, b, c$ and connected to the voltage source $U_{D}$. The positive pole of the voltage source $U_{D}$ is denoted as 1 while the negative one as 0 . Every switch is able to connect one pole of the source to the one phase output. According to this, switching states of the keys are also denoted by digits 1 and 0 respectively. The inverter state including states of all three keys can be described by using a set of digits $a b c$ where $a, b, c=0,1$. Of course the set of three elements where elements are represented by digits 0 or 1 may count eight variations that is: $000,001, \ldots 111$. It is useful and wide-spread in many papers to assume that these variations form binary numbers that is the numbers of the base- 2 positional numeral system. As a result each inverter state is defined by one three digit binary number where the order of digits corresponds to the order of phases $a b c$. Converting binary numbers to the decimal (base-10) number system it is possible to denote all eight inverter states by decimals $0,1_{10}, \ldots 7_{10}$ respectively. Thus the state denoted as $4_{10}(100)_{2}$ determines that the phase a is connected to the positive pole of the voltage source while the phases $b$ and $c$ - to the negative pole. The indices 2 and 10 indicate the base of the number system. This principle excluding decimal numbers will be used further in many examples and in other than binary number systems.

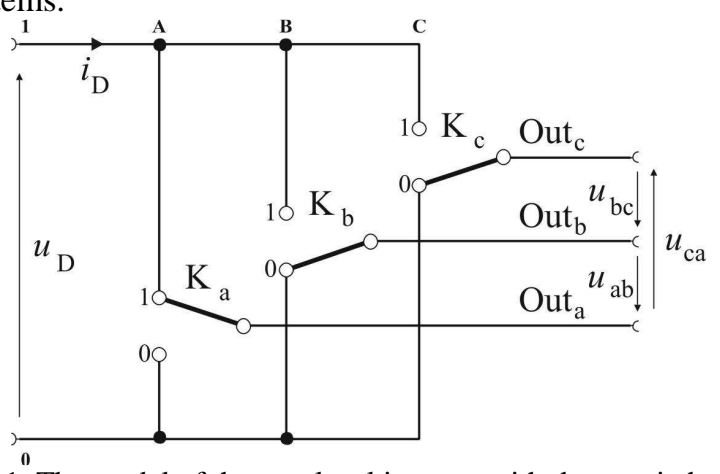

Fig. 1. The model of the two-level inverter with three switches.

The base- 2 and base- 10 number systems have been applied to circumscribe 2-level inverter states from the very beginning of the power electronics development. Although the rule of the precisely controlled relation "number-phase" has not always been kept. The abovementioned discussion has been cited in here since the idea of multilevel inverter circumscription will be based on further development of this concept.

\section{B. State-Vector of the 2-level Inverter}

The 2-level voltage source inverter (VSI) is able to produce eight different switching states. Successive states are denoted by index $\mathrm{k}=0,1, \ldots .7$. Any selected $k$-state means that three phase-to-phase voltages are connected to 
the load. These voltages form a column matrix of three elements thus defining the state vector $V_{k}$ of the VSI:

$$
V_{k}=\left[\begin{array}{l}
u_{a b_{k}} \\
u_{b c_{k}} \\
u_{c a_{k}}
\end{array}\right] \quad k=0,1, \ldots 7
$$

In a 2-level VSI the phase-to-phase voltages are formed as a potential difference between respective outputs. The switch $K_{a, b, c}$ can connect the output to the positive or negative pole of the voltage source $U_{D}$ according to the selected vector $V_{k}$.

The vector index $k$ can be reconverted to the binary numeral system and written as:

$$
k=\left(a_{k} b_{k} c_{k}\right)_{2} \quad \text { where } \quad a_{k}, b_{k}, c_{k}=0,1 \quad k=0,1, \ldots 7
$$

The binary expansion of the index $k$ permits to associate directly the phase output potential with the relevant binary symbol so it makes possible to determine output polar voltages which are referenced to the negative pole of the voltage source:

$$
u_{a 0_{k}}=a_{k} U_{D} \quad u_{b 0_{k}}=b_{k} U_{D} \quad u_{c 0_{k}}=c_{k} U_{D}
$$

As a result it permits to define the 2-level VSI state vector by use of respective binary symbols of the index $k$

$$
V_{k}=\left[\begin{array}{l}
u_{a b k} \\
u_{b c k} \\
u_{c a k}
\end{array}\right]=\left[\begin{array}{l}
u_{a o_{k}}-u_{b 0_{k}} \\
u_{b o_{k}}-u_{c 0_{k}} \\
u_{c 0_{k}}-u_{a o_{k}}
\end{array}\right]=U_{D}\left[\begin{array}{l}
\left(a_{k}-b_{k}\right)_{2} \\
\left(b_{k}-c_{k}\right)_{2} \\
\left(c_{k}-a_{k}\right)_{2}
\end{array}\right] \quad k=0,1, \ldots 7
$$

Since symbols $a_{k}, b_{k}, c_{k}$ may assume only values 0 or 1 than the output phase-to-phase voltage can assume only three values: $0,+U_{D},-U_{D}$.

\section{Delta Connected Load of the 2-level Inverter}

The inverter state vector $V_{k}$ operates on physical quantities and it needs no transformation to be utilized for calculation of the load current. A model of the 2-level inverter with a delta connected load is presented in Fig. 2. The inverter state determined by the vector $V_{k}$ (3) means that three phase-to-phase voltages are connected to the load.

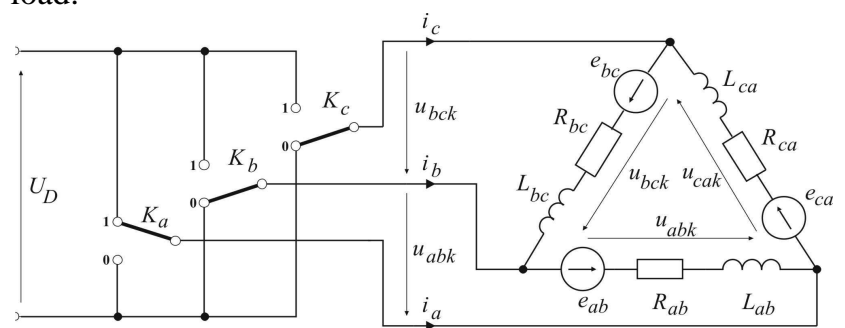

Fig. 2. The model of the two-level inverter with the delta connected load.

When the load is in delta connection than adequate phaseto-phase voltages are equal to the voltage given in (4). For any vector $V_{k}$ switched in time point $\mathrm{t}=\mathrm{t}_{\mathrm{n}}$ the inverter model reduces to the equivalent circuit described by the set of equations: $u_{a b_{k}}=R_{a b} \cdot i_{a b}(t)+L_{a b} \frac{d i_{a b}(t)}{d t}+e_{a b}(t)$

$\left.u_{b c_{k}}=R_{b c} \cdot i_{b c}(t)+L_{b c} \frac{d i_{b c}(t)}{d t}+e_{b c}(t)\right\}$

$u_{c a_{k}}=R_{c a} \cdot i_{c a}(t)+L_{c a} \frac{d i_{c a}(t)}{d t}+e_{c a}(t)$

Assuming counter RMF $e_{a b}, e_{b c}$ and $e_{c a}$ constant in a time interval $t\left\langle t_{n}, t_{n+1}\right\rangle$ and equal $E_{a b}, E_{b c}$ and $E_{c a}$ respectively it is simple to solve the equations and find expressions describing load currents (6). The symbol $\tau$ denotes the time constant of the one load leg and $I_{0 x x}$ current value in the time point $t_{n}$. The expressions (6) are valid in the aforesaid time interval between successive switching of the inverter states $V_{k(n)}$ and $V_{k(n+1)}$. The time interval is assumed to be very short in comparison to the time constant of the load circuit and expected period of the sine electromotive force.

$\left[\begin{array}{l}i_{a b}(t) \\ i_{b c}(t) \\ i_{c a}(t)\end{array}\right]=\left[\begin{array}{l}u_{a b k}-E_{a b} \\ u_{b c k}-E_{b c} \\ u_{c a k}-E_{c a}\end{array}\right] \frac{\left(1-e^{-\frac{t}{\tau}}\right)}{R}+\left[\begin{array}{c}I_{0 a b} \\ I_{0 b c} \\ I_{0 c a}\end{array}\right] e^{-\frac{t}{\tau}} \quad k=0,1, \ldots 7$

The expressions (4) and (6) define phase-to-phase voltages and load currents using corresponding symbols of the vector index $k$ binary expansion.

\section{Star Connected Load of the 2-level Inverter}

The 2-level inverter model with the load arrangement in a star connection is presented in Fig. 3. For the considered class of inverters it is possible to assume three equivalent definitions of the 2-level inverter state vector $V_{k}$.

$$
\begin{gathered}
V_{k}=U_{D}\left[\begin{array}{l}
\left(a_{k}-b_{k}\right)_{2} \\
\left(b_{k}-c_{k}\right)_{2}
\end{array}\right] \quad V_{k}=U_{D}\left[\begin{array}{c}
\left(b_{k}-c_{k}\right)_{2} \\
\left(c_{k}-a_{k}\right)_{2}
\end{array}\right] \\
V_{k}=U_{D}\left[\begin{array}{l}
\left(a_{k}-b_{k}\right)_{2} \\
\left(c_{k}-a_{k}\right)_{2}
\end{array}\right]
\end{gathered}
$$

in view of the fact that $u_{a b_{k}}+u_{b c_{k}}+u_{c a_{k}}=0$.

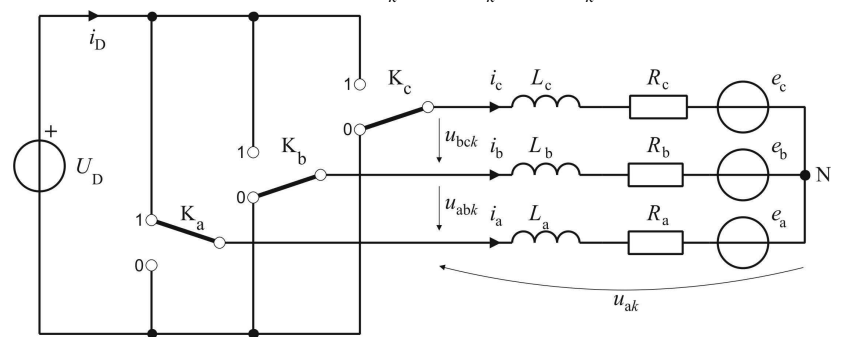

Fig. 3. The model of the two-level inverter with the star connected load.

For any vector $V_{k}$ switched on in the time point $\mathrm{t}=\mathrm{t}_{\mathrm{n}}$ the inverter model reduces to an equivalent two closed loop circuit presented in Fig. 4. Mathematically the circuit is described by the system of equations (8). 


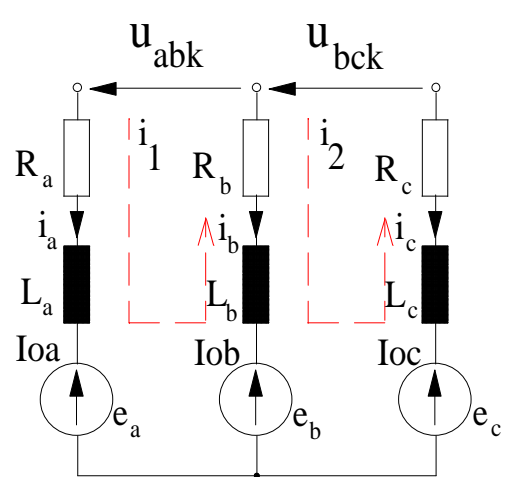

Fig. 4. The model of the two-level inverter with the star connected load.

$u_{a b k}=R_{a} \cdot i_{1}(t)+L_{a} \frac{d i_{1}(t)}{d t}+e_{a}(t)-e_{b}(t)+L_{b} \frac{d\left[i_{1}(t)-i_{2}(t)\right]}{d t}$

$+R_{b}\left[i_{1}(t)-i_{2}(t)\right]$

$u_{b c k}=R_{b}\left[i_{2}(t)-i_{1}(t)\right]+L_{b} \frac{d\left[i_{2}(t)-i_{1}(t)\right]}{d t}+e_{b}(t)-e_{c}(t)$

$+L_{c} \frac{d i_{2}(t)}{d t}+R_{c} i_{2}(t)$

Taking into consideration the relation between phase and loop currents:

$i_{\mathrm{a}}(t)=i_{1}(t), \quad i_{\mathrm{b}}(t)=i_{2}(t)-i_{1}(t), \quad i_{\mathrm{c}}(t)=-i_{2}(t)$

and introducing substitution

$2 U_{\mathrm{ab}_{\mathrm{k}}}+U_{b c_{\mathrm{k}}}=3 U_{a_{\mathrm{k}}}$

$U_{\mathrm{bc}_{\mathrm{k}}}-U_{a b_{k}}=3 U_{b_{k}}$

$-U_{\mathrm{ab}_{\mathrm{k}}}-2 U_{b c_{k}}=3 U_{c_{k}}$

one can obtain expressions describing the phase voltages $U_{a k}, U_{b k}, U_{c k}$ and phase load currents $i_{a}, i_{b}, i_{c}$. They all may be circumscribed using binary symbols of the vector index $k=\left(a_{k} b_{k} c_{k}\right)_{2}$ binary representation.

$$
\begin{aligned}
& {\left[\begin{array}{c}
U_{a k} \\
U_{b k} \\
U_{c k}
\end{array}\right]=\frac{U_{D}}{3}\left[\begin{array}{c}
2 a_{k}-b_{k}-c_{k} \\
2 b_{k}-a_{k}-c_{k} \\
2 c_{k}-a_{k}-b_{k}
\end{array}\right]} \\
& {\left[\begin{array}{l}
i_{\mathrm{a}}(t) \\
i_{\mathrm{b}}(t) \\
i_{\mathrm{c}}(t)
\end{array}\right]=\left[\begin{array}{l}
u_{a k}-E_{\mathrm{a}} \\
u_{\mathrm{b} k}-E_{\mathrm{b}} \\
u_{\mathrm{c} k}-E_{\mathrm{c}}
\end{array}\right] \frac{\left(1-\mathrm{e}^{-\frac{t}{\tau}}\right)}{R}+\left[\begin{array}{c}
I_{0 \mathrm{a}} \\
I_{0 \mathrm{~b}} \\
I_{0 \mathrm{c}}
\end{array}\right] \mathrm{e}^{-\frac{t}{\tau}}}
\end{aligned}
$$

The expressions are valid in a time interval $\mathrm{t}_{\mathrm{n}} \leq \mathrm{t} \leq \mathrm{t}_{\mathrm{n}+1}$ and under the same assumption as in previous example that is: counter EMF $e_{a}, e_{b}$ i $e_{c}$ is constant and equal to $E_{a}, E_{b} E_{c}$.

\section{E. Space Vector of the 2-level Voltage Source Inverter}

The switching $k$-state of the 2-level VSI generates three polar voltages $u_{a 0 k}, u_{b 0 k}, u_{c 0 k}$. They may be expressed on a complex plane by use of a transformation to the coordinate $\alpha \beta$-plane spanned by the $\alpha$-real axis (Realis) and the $\beta$-imaginary axis (Imaginaris). The commonly used space vector definition is the following
$\bar{V}_{k}=\frac{2}{3}\left(u_{a 0_{k}}+u_{b 0_{k}} e^{j(2 \pi / 3)}+u_{c 0_{k}} e^{-j(2 \pi / 3)}\right)$

The transformation result is called the voltage space vector and is a complex number which can be represented by the modulus $M$ and the argument (phase) $\varphi$

$$
\overline{V_{k}}=M_{k} e^{j \varphi_{k}} \quad M_{k}=\frac{2}{3} U_{D} \quad \varphi_{k}=\arccos \frac{\operatorname{Re}\left\{\bar{V}_{k}\right\}}{M_{k}}
$$

Introducing the equality (3) into (13) the definition of the vector can be written as follows

$$
\overline{V_{k}}=\frac{1}{3} U_{D}\left[\left(2 a_{k}-b_{k}-c_{k}\right)+j \sqrt{3}\left(b_{k}-c_{k}\right)\right]
$$

This equation expresses the space vector definition in symbols of the index $k$ binary expansion where $k$ is a decimal number discriminating the state vector.

The modulus value and the phase $\varphi$ of the space vector can be also expressed applying the symbols in question

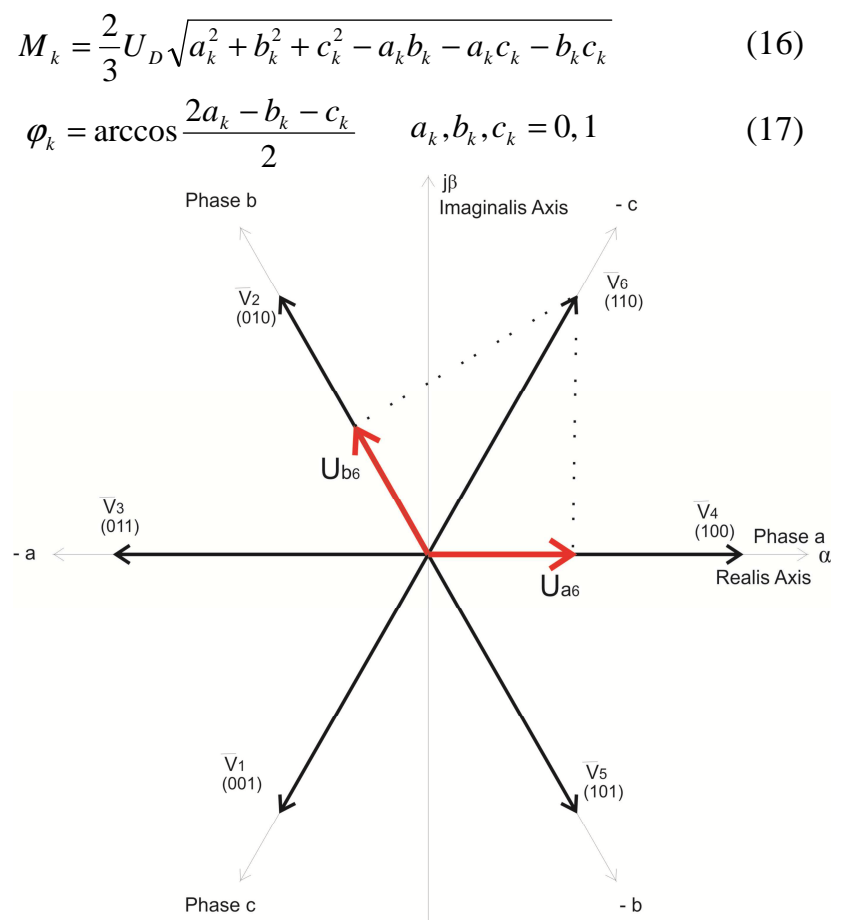

Fig. 5. Space vectors of the 2-level VSI.

All space vectors of the 2-level VSI have the same and equal to $2 U_{D} / 3$ modulus and are shifted each other on 60 degrees $(\pi / 3)$. Nevertheless the generalized expressions (16) and (17) will be useful in further discussion concerning multilevel inverters. The coefficient $2 / 3$ in (13) has been somewhat arbitrarily chosen although there are also reasons to use the value $\sqrt{2 / 3}$. There is no place here to discuss this problem but the assumed value of $2 / 3$ will be afterwards justified. All active space vectors are presented in Fig. 5. It is important to state here that definitions (13) and (15) relate to the space vector of the polar voltages $u_{a 0}, u_{b 0}, u_{c 0}$ but not to phase voltages. Applying the space vector of polar voltages to determine phase output voltages is evident and follows from simple projection of the selected vector on the phase axis. For 
instance the projection of the vector $\bar{V}_{6=(110)_{2}}$ to respective phases results in the following phase voltages:

$u_{a_{6}}=\left|\overline{V_{6}}\right| \cos (\pi / 3)=\frac{1}{3} U_{D}, \quad u_{b_{6}}=\left|\overline{V_{6}}\right| \cos (\pi / 3)=\frac{1}{3} U_{D}$

$u_{c_{6}}=\left|\overline{V_{6}}\right| \cos (-\pi)=-\frac{2}{3} U_{D}$

and are in accordance with (11) and expected physical evaluations. These values justify the usefulness of the assumed coefficient value $2 / 3$ in the space vector definition. Generally phase voltages are given as follows

$$
u_{a, b, c_{k}}=M_{k} \cos \varphi_{a, b, c_{k}}
$$

where the phase $\varphi$ is the angle between selected $k$-vector and $a, b, c$ axes. The result of (18) has to be in accordance with the expression (11).

\section{F. Phase-to-phase Voltage Space Vector of the 2-level VSI}

The transformation (13) can be applied to define the phase-to-phase voltage space vector. Its definition is the following

$$
\bar{V}_{p p k}=\frac{2}{3}\left(u_{a b_{k}}+u_{b c_{k}} e^{j(2 \pi / 3)}+u_{c a_{k}} e^{-j(2 \pi / 3)}\right)
$$

Introducing the equality (4) into (19) the definition of the space vector can be rewritten by use of the index $k$ binary symbols

$$
\bar{V}_{p p k}=U_{D}\left[\left(a_{k}-b_{k}\right)+j \frac{\sqrt{3}}{3}\left(a_{k}+b_{k}-2 c_{k}\right)\right]
$$

The modulus $M_{L L}$ and the argument $\varphi$ of the space vector can be also determined using these symbols

$$
\begin{aligned}
& M_{p p k}=\frac{2 \sqrt{3}}{3} U_{D} \sqrt{a_{k}^{2}+b_{k}^{2}+c_{k}^{2}-a_{k} b_{k}-a_{k} c_{k}-b_{k} c_{k}} \\
& \varphi_{k}=\arccos \frac{\sqrt{3}\left(a_{k}-b_{k}\right)}{2} \quad a_{k}, b_{k}, c_{k}=0,1
\end{aligned}
$$

All active space vectors $\bar{V}_{p p k}$ of the 2-level inverter have the same modulus measure, equal to

$$
M_{p p k}=\frac{2 \sqrt{3}}{3} U_{D}
$$

The vectors are presented in Fig. 6. They are shifted each other on 60 degrees $(\pi / 3)$ but no one lies on the Realis coordinate. The vector $\bar{V}_{p p 4=(100)_{2}}$ is shifted on 30 degrees ( $\pi / 6)$ to the $\alpha$ axis what indicates the phase lag of the phase-to-phase voltage $u_{a b}$ in relation to the phase voltage $u_{a}$. The modulus $M_{p p k}$ of the space vector is greater than $U_{D}$ voltage but its projection on two respective phase axes is equal to $U_{D}$. The projection on the third one axis is zero-dimensional because each one vector is perpendicular to one of phase axes. So it is possible to determine real phase-to-phase voltages of the selected space vector $\bar{V}_{p p k}$.

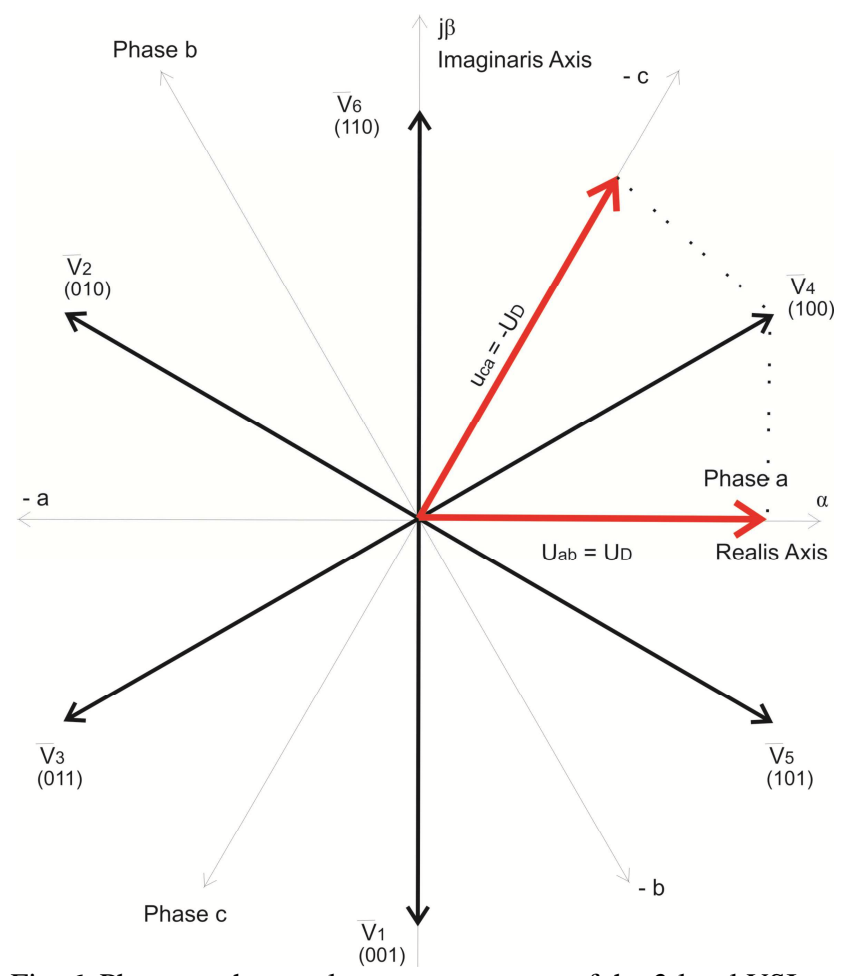

Fig. 6. Phase-to-phase voltage space vectors of the 2-level VSI.

For instance the projection of the vector $\bar{V}_{p p 4}=(100)_{2}$ to respective phases results in the following phase-tophase voltages:

$$
\begin{aligned}
& u_{a b_{4}}=\left|\bar{V}_{p p 4}\right| \cos (\pi / 6)=U_{D}, \quad u_{b c_{4}}=\left|\bar{V}_{p p 4}\right| \cos (\pi / 2)=0 \\
& u_{c a_{4}}=\left|\bar{V}_{p p 4}\right| \cos (\pi / 6)=-U_{D} \quad k=4
\end{aligned}
$$

and are in accordance with (4) and expected physical evaluations. These values have been obtained by use of the assumed coefficient value $2 / 3$ in the space vector definition. Generally phase-to-phase voltages are given as follows

$\left[\begin{array}{l}u_{a b_{k}} \\ u_{b c_{k}} \\ u_{c a}\end{array}\right]=M_{p p k}\left[\begin{array}{l}\cos \left(\varphi_{k}-\varphi_{a}\right) \\ \cos \left(\varphi_{k}-\varphi_{b}\right) \\ \cos \left(\varphi_{k}-\varphi_{c}\right)\end{array}\right]$

where $\varphi_{k}$ denotes the angle between selected vector $k$ and Realis axis $\alpha$ while $\varphi_{a, b, c}$ - the angle between vector $k$ and respective phase $a, b, \mathrm{c}$ axes. In the 2-level inverter all these angles can only assume values equal to multiple of 30 degrees so the cosine values are well known but this generalization of circumscription will be applied in further discussion.

\section{Conclusions}

The complexity of systems based on two and multilevel converters' structures, number of semiconductor switches and reachable vectors lead to use of extremely sophisticated control methods and algorithms. This requires availability of good mathematical tools and converter models which are useful to the purpose of control algorithm designing and guarantee easy implementation and fast performance of the converter. In power electronics domain there are a lot 
of proposals concerning converter models, notation systems and mathematical tools that authors use to apply.

The main goal of the contribution is to present some simplest mathematical system of notation and formulas. The converter may be described by use of defined state vector or classic space vector transform. The paper presents how to describe the converter states including two level inverters but the method could be developed to multilevel inverters. Thanks to its construction the system presents an easy to use mathematical tool. Using this tool it is possible to calculate available phase and phase-tophase voltage, state and space vectors and resulting in load currents.

\section{References}

[1] Iwaya K., Takahashi I.: Novel Multilevel PWM Wave Control Method using Series Connected Full Bridge Inverters, Proceedings of the IEEE Electric Machines and Drives Conference, volume 3, pages 1543-1548, June 2003.

[2] Faranda R., Valade I.: UPQC Compensation Strategy and Design Aimed at Reducing Losses. IEEE International Symposium on Industrial Electronics ISIE 2002, vol. 4, pp. 1264-1270.

[3] Sten K., Stacey E.: UPFC-Unified Power Flow Controller: Theory, Modeling, and Applications, IEEE Transactions on Power Delivery. Vol. 13. No. 4. October 1998.

[4] Kaźmierkowski M., P., F. Blaabjerg F.: Impact of Emerging Technologies on PWM Control of Power Electronics, IEEE Industrial Electronics Society Newsletter, December, 1995.

[5] Rodriguez J., Correa P., Moran L.: A vector control technique for medium voltage Multilevel Inverters, Proc. IEEE APEC, Anaheim, CA, pp. 173-178, March 2001.

[6] A.K. Gupta, A.M. Khambadkone: A General Space Vector PWM Algorithm for Multilevel Inverters, Including Operation in Over-modulation Range, IEEE Transactions on Power Electronics, vol. 22, no. 2, pp. 517-526, March 2007.
[7] Hashad M., Iwaszkiewicz J.: A Novel Orthogonal-VectorsBased Topology of Multilevel Inverters, IEEE Transactions on Industrial Electronics, vol. 49, no. 4, pp. 868-874, August 2002.

[8] Kawabata Y., Nasu M., Nomoto T., Ejiogu E.C., Kawabata T.: High-Efficiency and Low Acoustic Noise Drive System Using Open-Winding AC Motor and Two Space-VectorModulated Inverters," IEEE Transactions on Industrial Electronics, vol. 49, no. 4, pp. 783-789, August 2002.

[9] Iwaszkiewicz J.: A Novel Recurrent Approach to the Output Voltage Filtering of the Inverter, Electrical Power Quality and Utilization, Polish Academy of Science, Volume 9, No. 1, September 2003, pp. 59-65.

[10] M. Malinowski, K. Gopakumar, J. Rodriguez, M.A. Perez: A Survey on Cascaded Multilevel Inverters, IEEE Transactions on Industrial Electronics, vol. 57, no. 7, pp. 2197-2206, July 2010.

[11] Escalante M.F., Vannier J-C., Arzande A.: Flying Capacitor Multilevel Inverters and DTC Motor Drive Applications, IEEE Transactions on Industrial Electronics, vol. 49, no. 4, pp. 809-815, August 2002.

[12] P. Lezana , J. Pou, T.A. Meynard, J. Rodriguez, S. Ceballos, F. Richardeau: Survey on Fault Operation on Multilevel Inverters, IEEE Transactions on Industrial Electronics, vol. 57, no. 7, pp. 2207-2218, July 2010.

[13] M.B. Smida, F.B. Ammar: Modeling and DBC-PSC-PWM Control of a Three-Phase Flying-Capacitor Stacked Multilevel Voltage Source Inverter, IEEE Transactions on Industrial Electronics, vol. 57, no. 7, pp. 2231-2239, July 2010.

[14] B. Vafakhah, J. Salmon, A.M. Knight: A New Space-Vector PWM with Optimal Switching Selection for Multilevel Coupled Inductor Inverters, IEEE Transactions on Industrial Electronics, vol. 57, no. 7, pp. 2354-2364, July 2010.

[15] Celanovic N., Boroyevich D.: A Fast Space-Vector Modulation Algorithm for Multilevel Three Phase Converters, IEEE Transactions on Industry Applications, vol. 37, no. 2, pp. 637-641, March/April 2001. 